\title{
Review of: "Fractional-order LCL filters: principle, frequency characteristics, and their analysis"
}

roberto lojacono ${ }^{1}$

1 University of Rome Tor Vergata

Potential competing interests: The author(s) declared that no potential competing interests exist.

In the article, the authors insert components with fractional cons

New Peer Review

Qeios ID: ZGC34I

Review of: Fractional-order LCL filters: principle, frequency characteristics, and their analysis

roberto lojacono $^{1}$

Author(s) details

In the article, the authors insert components with fractional constitutive equations in the LCL filters. These analog filters, which are inserted in the UPS circuits, are currently still being developed unlike the others analog ones that have lost importance with the advent of digital circuits. The insertion of fractional components provides some additional parameters which, in theory, should allow a greater possibility of adapting the characteristics of the filters themselves to the specific requests.

However, the insertion of these components makes the analysis of these networks more complicated and, consequently, also the synthesis. The authors carry out the analysis of these networks in the Laplace domain and use the formulation of the L-transformed according to Caputo for the transformation of the characteristics of the fractional components. Some authors have highlighted differences between the results of this transformation and those obtained instead following the dictates of Riemann-Lioville. The authors do not mention these deviations which could however be of little importance from a practical point of view. In this view , Caputo's transformation appears to be the most convenient. It allows, with zero initial conditions, to proceed with the analysis with the same techniques used for non-fractional circuits: the analysis of the latter becomes a special case of "fractional" analysis.

The authors carry out the analysis of the LCL structures thus modified and present a complete examination of the properties of the filters thus obtained, especially as regards the resonance frequencies, which are critical for the filtering action. They also present feedback with simulations that show the intrinsic correctness of the analysis carried out and the properties obtained. The results therefore appear to be very 
useful for the designers of these circuits.

Recommendations for the authors:

- formula cited in the introduction, is declared incorrect but appear coherent with formula (17), here developed when the total fractional order is 2. Make this point more clear.

- Ref. 6 seems to be incorrect. 